\title{
Chief Editor Introduction
}

Volume VIII Issue 2 of the Journal of Muslim Mental Health features two articles that describe how young Muslims may acculturate in Western societies. The first, by Goforth et al, describes the role of acculturation, acculturative stress and religiosity in psychological adjustment using well validated psychometic tests. As expected, and seen in other ethnic and religious minorities, the longer theses Arab-American Muslim subjects lived in the U.S. the higher their acculturation scores were. Furthermore, subjects who scored higher on religiosity scores reported less acculturation. However, the interesting finding is the negative association between religiosity and experiencing discrimination. In other words, subjects who reported higher religiosity scores reported experiencing less stress from discrimination. While the study did not account for variables such as socio-economic status and access to resources and activities, the potential role of religiosity as a protective factor from experiencing stress from discrimination warrants further exploration.

Jaimee Stuart, in the second article of the journal's issue, examined the experience of New Zealand Muslim adolescents by conducting in-depth interviews and focus group analyses. The subjects from this study were recruited from a Muslim student organization, came from diverse ethnic backgrounds, but appeared (although it was not reported explicitly) to be almost if not all immigrants. Subjects in this article reported that they successfully navigated the stress of living bicultural lives by drawing from both family support and their religious belief. In contrast to Gofort et al's article, the subjects' narratives describe how their Islamic practice and identity is a source of both stress, especially for women who wear the headscarf, and strength. Given that subjects were recruited from a Muslim student organization, they are more likely to draw strength and gratification from their religious activism than other Muslim adolescents.

Our third article offers another measure of spirituality in the Muslim context. In any research that explores mental health and psychological function in the Muslim communities, religiosity and spirituality are critical variables to explore. Religiosity and spirituality represent two different constructs, with the former often describing connection and adherence to particular religious doctrine while the later connotes transcendent values or feelings outside the mate- 
rial world. A challenge in measuring spirituality in the Muslim specific context is that features, and particularly rituals, within Islam are based on religious doctrine. While the Multidimensional Measure of Islamic spirituality includes many items centered on religious practices, it emphasizes self-discipline, faith, and connectedness and may serve as an important scale for future research in the field.

This issue of journal ends with four abstracts, selected from 20 , which were presented at the $6^{\text {th }}$ Annual Muslim Mental Health Conference hosted by the Michigan State University Department of Psychiatry. The work illustrates the exciting, emerging research on Muslim mental health globally. 